\title{
Genetic mapping and manipulation: Chapter 7-Making compound mutants ${ }^{*}$
}

\author{
David Fay ${ }^{\S}$, Department of Molecular Biology, University of Wyoming, \\ Laramie, Wyoming 82071-3944 USA
}

\section{Table of Contents}

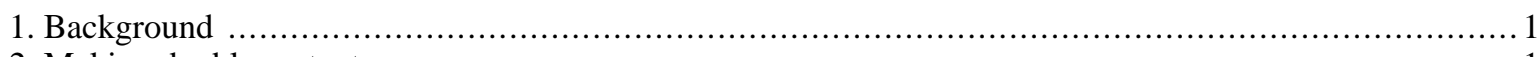

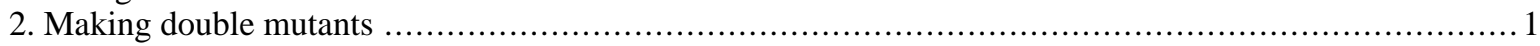

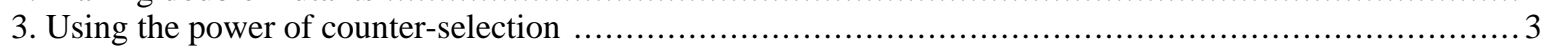

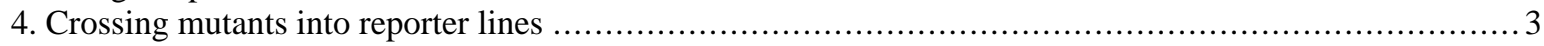

\section{Background}

One of the most important genetic skills is the ability to generate double- and triple-mutant strains for phenotypic and genetic analysis. These compound mutants can be used for epistasis experiments for ordering genes within a genetic pathway or for identifying genetic interactions such as Suppressor mutations or Synthetic and enhancer mutations of a given phenotype. Outlined below are a few of the basic methods used for this purpose.

\section{Making double mutants}

In the simplest case, two mutations ( $\boldsymbol{a}$ and $\boldsymbol{b}$ ) are unlinked, viable, and associated with an easily discernable plate phenotype. In this situation, it is a straightforward matter to cross one of the two strains to N2 males (or to generate a homozygous male stock from one of the mutant strains), and then cross the resultant heterozygous males to the second mutation to generate the $\boldsymbol{a} / \mathbf{+} ; \boldsymbol{b} / \mathbf{+}$ trans-heterozygote. A B animals (1/16) can then easily be isolated in the next generation (Figure 1; strategy \#1). In cases where $\boldsymbol{a}$ or $\boldsymbol{b}$ mutations do not produce an obvious phenotype or perhaps produce highly similar phenotypes, it may be highly advantageous to have one or both mutations linked to a nearby genetic marker (e.g., dpy or $\boldsymbol{u n c}$ ). In this case, $\boldsymbol{a} \boldsymbol{u} \boldsymbol{u} \boldsymbol{c} /+$ males might be crossed to $\boldsymbol{d p y} \boldsymbol{b}$ hermaphrodites to generate $\boldsymbol{a}$ unc/+; dpy $\boldsymbol{b} /+$ trans-heterozygotes. Following self-fertilization, Dpy Unc progeny of the desired phenotype ( $\boldsymbol{a}$ unc; $\boldsymbol{d} \boldsymbol{p y} \boldsymbol{b}$ ) can be isolated (Figure 1; strategy \#2). It is important to keep in mind that when using linked markers to follow mutations of interest, the distance between the mutation and the visible marker will strongly affect the reliability of the approach. Namely, the farther apart the mutation is from the visible marker, the more likely that a recombination event might lead to the mutant allele becoming separated from the marker. Thus it is always essential to generate several independent lines and to confirm the correct genotype either by direct sequencing of the mutant locus or by obtaining consistent results for multiple independent isolates.

\footnotetext{
*Edited by Victor Ambros. Last revised May 4, 2005. Published February 17, 2006. This chapter should be cited as: Fay, D. Genetic mapping and manipulation: Chapter 7-Making compound mutants (February 17, 2006), WormBook, ed. The C. elegans Research Community, WormBook, doi/10.1895/wormbook.1.96.1, http://www.wormbook.org.

Copyright: () 2006 David Fay. This is an open-access article distributed under the terms of the Creative Commons Attribution License, which permits unrestricted use, distribution, and reproduction in any medium, provided the original author and source are credited.

${ }^{\S}$ To whom correspondence should be addressed. E-mail: davidfay@uwyo.edu
} 
\#1

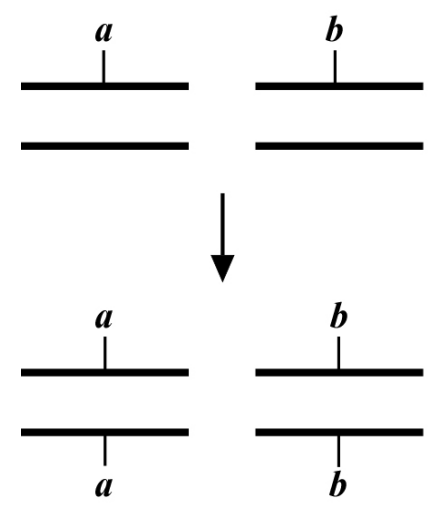

A B

1/16
\#2

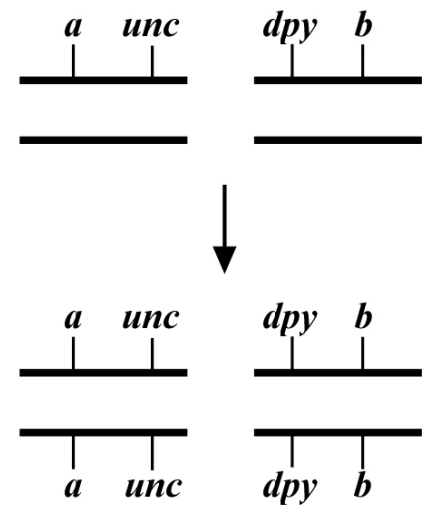

"A" Unc Dpy "B"

1/16 - not including recombinants

Figure 1.

The real difficulty with creating compound mutants comes when two of the mutations are on the same chromosome. In this situation, one can either take a passive or active approach. An example of the passive approach is shown in Figure 2. In essence, you create a trans-heterozygote strain where the mutations of interest are balanced by each other. Then by blindly picking sufficient animals of either mutant genotype, you can hope to isolate a low percentage of animals that throw the double-mutant chromosome as a result of a crossover event. This can be done either with or without the use of linked markers, depending on the phenotype of the individual mutants. This approach is quite effective if the two mutations aren't too closely linked and has the advantage of not necessarily requiring any special strain construction beforehand.

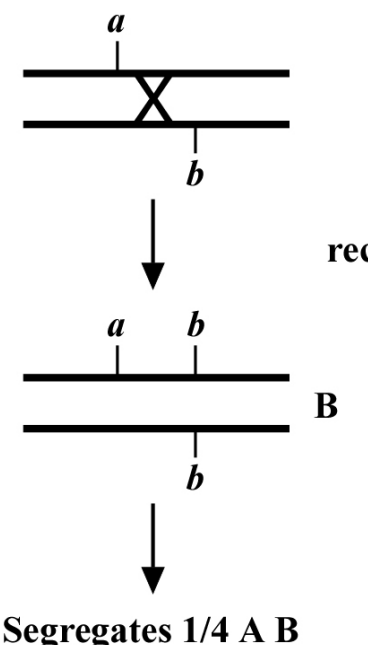

Segregates 1/4 A B

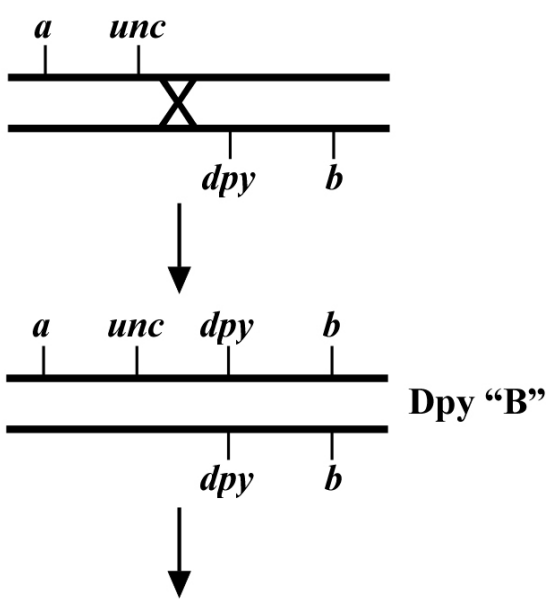

Segregates 1/4 "A" Unc Dpy "B"

Figure 2.

Alternatively, if the two mutations are very closely linked, you may find it difficult or impossible to isolate double-mutant animals of the desired genotype. Here it will be best to take an active approach. For this to be feasible, however, it will require a bit of prior strain construction. Fortunately, if you have previously generated a strain containing your mutation flanked by two visible markers (e.g., for SNP mapping), you should be in a good position to begin your crosses. In this case, you will want to carry out crosses to generate a strain such as $\boldsymbol{u n c} \boldsymbol{a}$ $\boldsymbol{d} \boldsymbol{p} \boldsymbol{y} / \boldsymbol{b}$, where $\boldsymbol{a}$ and $\boldsymbol{b}$ are the mutations you wish to link in cis and $\boldsymbol{u n c}$ and $\boldsymbol{d} \boldsymbol{p} \boldsymbol{y}$ are visible markers that flank $\boldsymbol{a}$ (Figure 3). Depending on the specific locations of the various mutations relative to each other, you will want to isolate either Unc-A (non-Dpy) or A Dpy (non-Unc) recombinant animals and then look for the presence of Unc A B or B A Dpy progeny in the next generation. This approach is best accomplished if $\boldsymbol{b}$ lies outside the region encompassed by $\boldsymbol{u n c}$ and $\boldsymbol{d p y}$; however, even if $\boldsymbol{b}$ is internal, strains of the desired genotype should almost always be obtainable if sufficient recombinants are analyzed. 

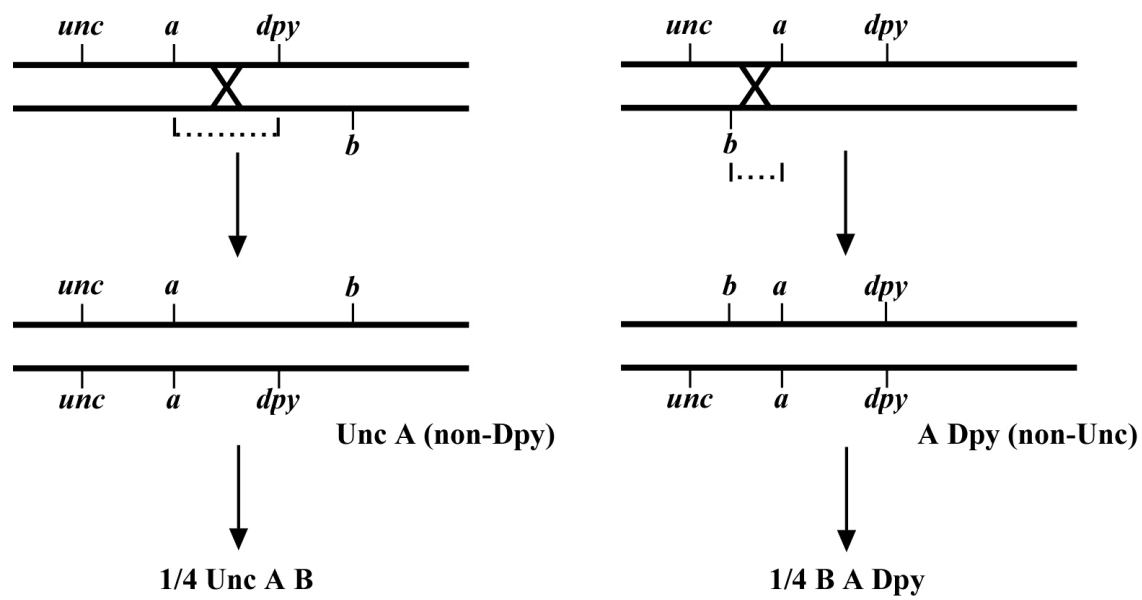

Figure 3.

\section{Using the power of counter-selection}

Another very useful trick when working with mutations that are difficult to track based on their plate phenotype is the use of the counter-selectable marker. There are many variations to this theme, two of which are outlined in Figure 4. In both cases, a $\boldsymbol{c} / \boldsymbol{c} ; \boldsymbol{m} / \boldsymbol{m}$ double mutant is sought. In case $\# 1, \boldsymbol{m} / \boldsymbol{m}$ animals display the phenotype $\mathbf{M}$, whereas $\boldsymbol{c} / \boldsymbol{c}$ mutants fail to display an obvious phenotype. Homozygous $\boldsymbol{c} / \boldsymbol{c}$ males are crossed to $\boldsymbol{m} / \boldsymbol{m}$; unc/unc hermaphrodites, where the unc mutation is located on the same chromosome and is preferably close to the genetic position of $\boldsymbol{c}$. In subsequent generations, the absence of Unc animals can be used to infer that $\boldsymbol{c}$ is probably homozygous. In case \#2, $\boldsymbol{m} / \boldsymbol{m}$ animals display no obvious defects, whereas $\boldsymbol{c} / \boldsymbol{c}$ animals display the $\mathbf{C}$ phenotype. Here, N2 males are first crossed to the Unc strain to generate heterozygous unc/+ males, which are subsequently crossed to c/c hermaphrodites. Males resulting from this latter cross (50\% of which will be unc/+; c/+) are then crossed to $\boldsymbol{m} / \boldsymbol{m}$ hermaphrodites, and resultant cross progeny of the genotype $\boldsymbol{c} /+; \boldsymbol{u n c} / \boldsymbol{m}$ are identified. Finally, $\mathbf{C}$ animals that fail to segregate Unc progeny are identified indicating the desired genotype. We also note that in addition to standard genetic markers, balancer chromosomes containing integrated GFP-expressing arrays can be exceptionally useful for counter selection. In fact, the dominant effect of the GFP means that homozygous $\boldsymbol{c} / \boldsymbol{c}$ animals (to site the above case) can be identified directly from the progeny of $\boldsymbol{c} / \boldsymbol{G F P}$ heterozygotes.

$\# 1$
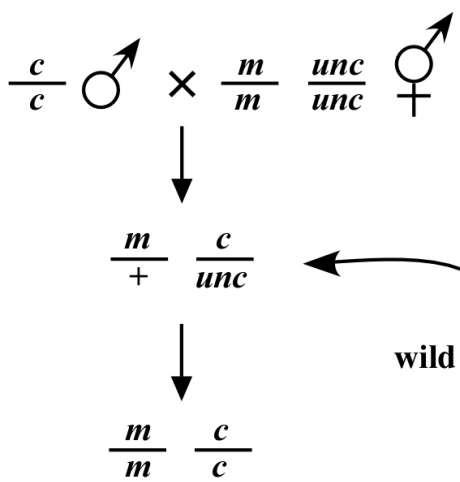

M C - does not segregate Uncs
\#2
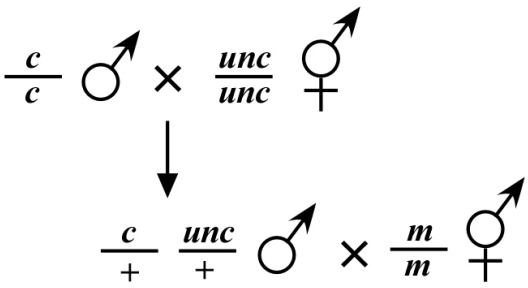

wild type - segregates Uncs
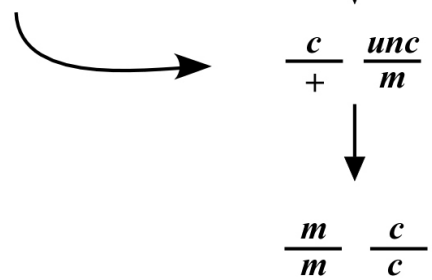

M C - does not segregate Uncs

Figure 4. 


\section{Crossing mutants into reporter lines}

All the principles described above will apply to the generation of mutant strains containing various integrated GFP or lacZ reporters. In the case of GFP, the dominance of the phenotype (green worms) makes these experiments quite straightforward to conduct. In fact, in many cases one can distinguish directly GFP/GFP and GFP/+ animals, as the former fluoresce more brightly than their heterozygous counterparts. One issue that you may encounter is that a number of integrated GFP lines also contain the dominant rol-6 marker. This may influence the way in which you design your crosses, as rolling males rarely mate well. In addition, there is an interesting phenomenon concerning males that carry an extrachromosomal array. Namely, some degree of mutual exclusion between the X chromosome and the array results in far fewer hermaphrodite cross progeny acquiring the array than might be expected. Thus, it may be necessary to compensate by setting up more mating plates than would be.

All WormBook content, except where otherwise noted, is licensed under a Creative 